\title{
Multiple-wavelength lasing in one-dimensional bandgap structures: implementation with active $n-i-p-i$ layers
}

\author{
Andrey G. Smirnov, Dmitrij V. Ushakov, and Valerii K. Kononenko \\ Stepanov Institute of Physics, National Academy of Sciences of Belarus, F. Scaryna Avenue, 70, \\ 220072 Minsk, Belarus
}

Received December 1, 2001; revised manuscript received April 4, 2002

\begin{abstract}
We study the optical localized states in a one-dimensional system of strongly coupled defect microcavities for the case when a tight-binding approximation is not valid. Transmission and electromagnetic mode density spectra as well as the distribution of light intensity inside the bandgap material are investigated. We report on the effect of splitting the fundamental coupled-cavity mode into several high- $Q$ submodes to support perfect transmission of light at low group-velocity values. New types of laser microcavities that provide lowthreshold lasing at multiple wavelengths and in opposite directions are proposed. Possible implementation of the laser systems with active $n-i-p-i$ layers is discussed. (C) 2002 Optical Society of America
\end{abstract}

OCIS codes: $140.3410,190.0190,310.0310$.

\section{INTRODUCTION}

In the era of rapid progress in wavelength-division multiplexing technologies, cost-effective supercompact multiple-wavelength lasers that can be used as light sources in all-optical integrated circuits are in great demand. At present, two-or three-dimensional photonic crystals as well as one-dimensional (1-D) bandgap structures are generally recognized as forming a natural foundation of future wavelength-division-multiplexing photonics. This is so because of the unique properties of these structures, such as spectral selectivity, high concentration of light intensity within small time-spatial volumes, and substantial decrease of the speed of light, resulting in alteration of the spontaneous-emission rate of embedded atoms or clusters ${ }^{1}$ and in gain enhancement. ${ }^{2,3}$ Based on photonic bandgap (PBG) engineering, low-threshold microlasers can be created.

The motivation for the present study comes from the results of recent investigations of $1-D$ bandgap structures, demonstrating that a dramatic enhancement of optical gain is attained when there is an appropriate arrangement of composite crystal geometry in spite of the fact that the amplification for a single active layer is not large. $^{2-4}$ This effect occurs at certain frequencies because of the existence of specific high- $Q$ modes, e.g., transmission resonance, ${ }^{4}$ defect, ${ }^{3}$ or coupled-cavity ${ }^{5}$ modes, in which light is slowed down to form localized states. We develop the basic ideas of gain enhancement in PBG material and search for a suitable 1-D laser microresonator layout to support a set of separate high- $Q$ lasing modes of comparable threshold. This permits the creation of supercompact light sources to provide either simultaneous or switched lasing at multiple wavelengths.

Also, in this paper we propose a PBG design that combines a wide range of possibilities for controlling the optical properties of doping semiconductor superlattices, e.g., dispersion, luminescence, absorption, and amplification spectra together with the unique ability of a bandgap material to concentrate electromagnetic energy in time and space. The doping superlattices, or $n-i-p-i$ crystals, belong to peculiar artificial tunable low-dimensional systems. ${ }^{6}$ Optical and electric properties of the superlattices can be varied in a wide range by excitation and through the choice of thickness and doping of the crystal layers. ${ }^{6,7}$ The main properties of these superlattices are (a) spatial separation of electrons and holes in different remote quantum wells, (b) energy bandgaps tunable by optical or electric excitation, (c) enhanced current carrier lifetime and decreased oscillator strength of optical transitions, (d) strong modification of the energy-level structure, depending on the distribution and concentration of doping impurities, and (e) wide variation of the properties and characteristics that are due to design parameters or to the introduction of additional quantum wells and $\delta$-doped regions. Based on these unique tunable properties of the doping superlattices, various compact photonic structures with $n-i-p-i$ layers can be designed for laser applications. (See Fig. 1.)

The paper is organized as follows: In Section 2 we describe some consequences of the linear theory of laser resonators and discuss our model and methodology. In Section 3 a new layout for 1-D bandgap structures is proposed that consists of a sequence of strongly coupled defect microcavities. We demonstrate that overcoming the tight-binding limit leads to the effect of splitting the eigenmode of a single microcavity into several defect modes, with each mode preserving its high- $Q$ properties. In Section 4 the feasibility of using photonic $n-i-p-i$ heterostructures for laser applications is shown. New types of bandgap microcavities are proposed that provide lowthreshold lasing at multiple wavelengths and in opposite directions. 
(a)

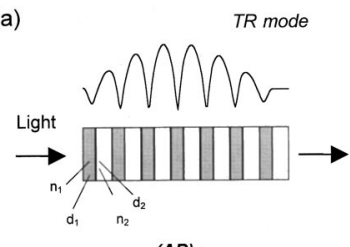

$(A B)_{7}$

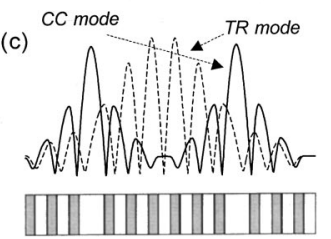

$\left\{(A B)_{3} D(A B)_{3}\right\}_{2}$

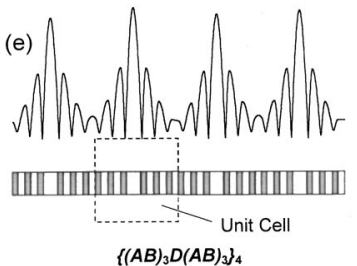

(b)

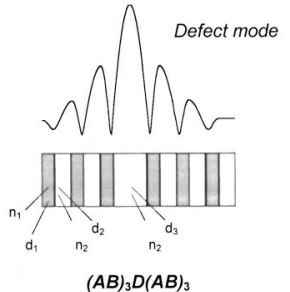

(d)

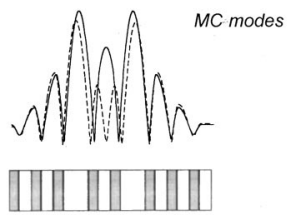

$(A B)_{3}\left\{D(A B)_{2} D\right\}(A B)$

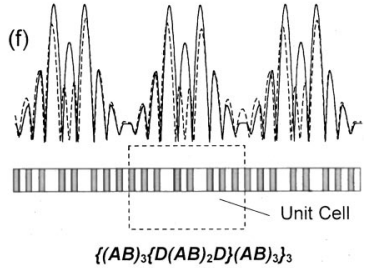

Fig. 1. High- $Q$ modes of various layouts of 1-D bandgap resonators: (a) generic periodic stack, (b) defect microcavity, (c) coupled-cavity resonator (CCR) consisting of two defect microcavities (weak coupling), (d) mixed-cavity-resonator (MCR) containing two microcavities (strong coupling), (e) four microcavities that form a CCR, (f) coupled MCR. TR, transmission resonance. See text for details.

\section{MODEL AND METHODOLOGY FOR STUDY OF PHOTONIC BANDGAP RESONATORS}

We deal with layered PBG material of length $L$ consisting of a finite number of slabs, each of them of a different thickness and with different optical properties. Our main goal is to develop a high- $Q$ microresonator that will support lasing at multiple wavelengths. We intend to consider various layouts of 1-D bandgap structures (see Fig. 1) and find out what type of lasing mode a certain layout exhibits, and what a threshold magnitude is. In this paper a specific notation is used for encoding various types of layered and, in particular, periodic structures. We denote by a character a unique layer that has a certain material type and thickness. Enclosing a set of characters in brackets defines an elementary cell that can be replicated to form a periodic arrangement of a bandgap material. For example, $(A B)_{40}$ corresponds to a twocomponent generic periodic stack that comprises 40 pairs. By defining original composition and multiplication rules we can write the equivalents, e.g., $(A B)_{40}=\left\{(A B)_{20}\right\}_{2}$ $=(A B)_{20}(A B)_{20}$.

Let us describe our preliminary model. We assume that some of layers of a PBG crystal are doped with an active material. Because we are interested only in resonator characteristics and not in lasing dynamics, the whole analysis can be done within the framework of linear theory. So the optical properties of layers are described by linear complex refractive index $\tilde{n}=n$ $+i g \omega^{-1} c / 2$, where gain coefficient $g$ and $n$ do not depend on the intensity of light. In the general case the quantities $n$ and $g$ are functions of radiation frequency $\omega$ $=2 \pi c / \lambda$ ( $\lambda$ and $c$ are, respectively, the light's wavelength and velocity in vacuum). For our purposes we consider only the case when light propagates along the axis of a bandgap structure ( $Z$ axis). Also, let us assume for simplicity that all active layers of our PBG laser are homogeneously pumped, i.e., have the same value of $g$.

One common procedure for determining a set of lasing modes frequencies $\omega_{m}$ and corresponding threshold values $g_{m}$ for a PBG structure with arbitrary spatial distribution of refractive index $\widetilde{n}(z)$ is to solve either of these equations:

$$
T(\omega, g)=\infty
$$

or

$$
R(\omega, g)=\infty,
$$

where $T=I_{t} / I_{0}$ and $R=I_{r} / I_{0}$ are transmission and reflection coefficients, respectively, of a structure $\left(I_{0}, I_{t}\right.$, and $I_{r}$ are correspondingly the intensity of incident, transmitted, and reflected radiation). Physically, Eqs. (1) mean that one has finite output intensity $I_{t, r} \neq 0$ at zero input signal $I_{0}=0$; i.e., the generation of light occurs. ${ }^{8}$

Equations (1) are barely resolved analytically for arbitrary layouts of PBG material, and usually some numerical approach is involved. Besides, to find out what PBG layout is more efficient for lasing, sometimes it is not necessary to obtain the exact values of $g_{m}$. Instead, what we need is the knowledge of which PBG layout provides the minimum threshold. We could obtain this information by examination of the spectrum of density of modes (DOM), $\rho(\omega)=V^{-1}(\omega)=\mathrm{d} \omega / \mathrm{d} K$; the physical quantity is inverse group velocity $V$. As was mentioned in Ref. 9, at frequencies corresponding to the high values of $\rho$ the following effects can be observed: (i) substantial decreasing of the group velocity of light inside PBG material, i.e., increase of optical pass, and (ii) spatial light localization, when the radiation intensity within the structure $\langle I(\omega)\rangle$ $=L^{-1} \int I(\omega, z) \mathrm{d} z$ grows on average. As is known, these effects, which result in optical gain enhancement, become apparent either at the transmission resonances near the edges of the forbidden gap $^{2,4}$ or at defect $\operatorname{modes}^{3}$ of a bandgap structure.

To get more information on the DOM we plot in a single figure $\rho(\omega)$ calculated for a totally passive ( $g=0$ ) generic periodic stack together with $T(\omega, g)$ contours obtained for the same stack with $g \neq 0$ (Fig. 2). As can be clearly seen, $T(\omega, g)$ tends to infinity at several frequencies that coincide with the transmission resonances of the passive structure. These are frequencies at which $\rho(\omega)$ attains its maxima. Also, the larger the value of the DOM, $\rho_{m}$ $=\rho\left(\omega_{m}\right)$, is, the lower the lasing threshold $g_{m}$ is for a certain mode. A similar relation among transmission, DOM, and lasing characteristics can be verified for arbitrary PBG layouts. Thus all the necessary qualitative information on lasing modes and thresholds can be obtained from DOM spectra at frequencies where $\mathrm{d} \rho / \mathrm{d} \omega=0$ and $\mathrm{d}^{2} \rho / \mathrm{d} \omega^{2}<0$. The latter of these approaches is expected to be much simpler than resolution of Eqs. (1).

We use the definition of the DOM proposed in Ref. 9: 


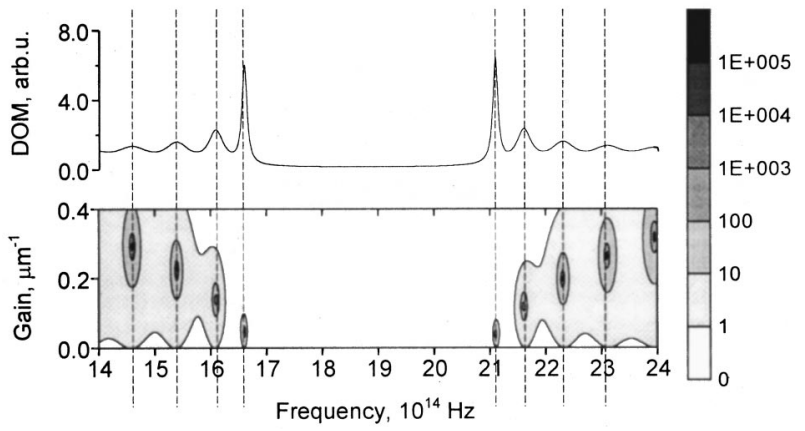

Fig. 2. Contours of transmission $T$ in plane $(\omega, g)$ and DOM spectrum $\rho(\omega)$ for the $(A B)_{20}$ stack. The parameters of the structure are $n_{1}=1.41, n_{2}=1.0, n_{1} d_{1}=n_{2} d_{2}=0.25$, and $g_{1}$ $=g_{2}=g$.

$$
\rho=L^{-1}\left(y^{\prime} x-x^{\prime} y\right) /\left(x^{2}+y^{2}\right),
$$

where $t=T^{1 / 2} \exp (i \varphi)=x+i y$ is the amplitude transmission coefficient. To calculate the transmission and the DOM we apply the well-known transfer-matrix method. ${ }^{10}$ The transfer-matrix method is an effective tool that is commonly used to simulate transmission and reflection spectra of layered inhomogeneous structures. More specifically, in our approach we employ the transfermatrix method for calculation of intensity $I(z)$ or amplitude $|E(z)|$ light patterns inside a bandgap material. Generally, three factors influence the efficiency of lasing in a PBG structure: (i) high values of gain $g(\omega)$ within a certain frequency range; (ii) large values of DOM $\rho(\omega)$; and (iii) perfect spatial matching of a light-intensity pattern with the distribution of gain within structure (i.e., the overlap integral $\int I(z) g(z) \mathrm{d} z$ has to be maximum ${ }^{2}$ ). Thus, knowledge of the field distribution is important and provides more physical insight into lasing performance.

In Section 3 we exploit the methodology described, i.e., calculation of the density spectra and interior light patterns of transmission and electromagnetic modes for investigation of high- $Q$ localized states of several layouts of PBG materials. In particular, 1-D systems of strongly coupled defect microcavities are studied.

\section{MIXED LOCALIZED MODES IN ONE- DIMENSIONAL COUPLED-CAVITY STRUCTURES}

Let us clarify more precisely what we expect from a multiple-wavelength PBG resonator. The result presented in Fig. 2 demonstrates that, naturally, exciting multiple lasing modes of $\{A B\}_{N}$ bandgap structure presents no problem. However, among the allowed frequencies, the number of high- $Q$ modes (with a minimum of $g_{m}$ and a maximum of $\rho_{m}$ ) is limited by two transmission resonance (TR) modes, $\omega_{ \pm 1}$, at the edges of the forbidden gap, and strong discrimination is observed for all other modes of the higher order. Moreover, such a resonator has a small number of degrees of freedom to vary the relative spectral positions of modes. Instead of TR modes, one may operate at the defect mode of a 1-D symmetric microcavity $\{A B\}_{J} D\{A B\}_{J}$, which is widely used in vertical-cavity surface-emitting lasers. It is well known that in such structures light is strongly localized in the vi- cinity of defect layer $D$. The calculated value of the DOM for the defect mode is much higher than the corresponding values of TR modes obtained for the generic stack at a fixed overall length of the structure. As was reported earlier, ${ }^{3}$ the result is stronger spatial localization and, hence, an order-of-magnitude smaller lasing threshold for the defect mode than for the transmission resonance mode. The only drawback of such microcavities is their single lasing frequency. So it would be desirable to have a resonator of simple and flexible design supporting multiple high- $Q$ lasing modes similar to the eigenmode of a defect microcavity.

Below, we propose a new layout of 1-D bandgap structure with the properties needed, which is based on the system of strongly coupled microcavities. We exploit the analogy of the wavelike effects in solid-state physics, electronics, and optics. In particular, it is known that the interaction between $N$ oscillators with eigenfrequency $\omega_{0}$ causes the splitting of $\omega_{0}$ into $N$ subfrequencies. Here two qualitatively different regimes have to be considered, i.e., strong and weak coupling. In the case of strong coupling the oscillating wave functions overlap significantly, forming new eigenmodes with frequencies $\omega_{0}{ }^{\prime}$. The closer the oscillators are packed and the stronger the interaction potential, the larger is the spectral shift $\Delta \omega^{\prime}$ $=\omega_{0}{ }^{\prime}-\omega_{0}$. That is drastically different from weak coupling, or coupling in a tight-binding (TB) limit, whose optical analog was recently described in Ref. 11. Following the TB scheme, the overlap of wave functions is small; as a result, eigenfrequency $\omega_{0}$ remains almost unchanged. The same is true for wave functions to which only small corrections are added.

In Fig. 1 we present sketchy layouts of bandgap structures that correspond to the various types of coupling of defect microcavities that form coupled-cavity resonators (CCRs). Using the notation offered in Section 2, we encode as $\left\{(A B)_{J} D(A B)_{J}\right\}_{N}$ a CCR consisting of $N$ weakly coupled microcavities [Figs. 1(c) and 1(e)]. In such a system a single microresonator represents a unit cell that may be translated $N$ times to form the whole structure. In Figs. 3(a)-3(c) one may track the changes in the transmission spectrum at the transition from a single unit cell to a CCR. In particular, with the increase of $N$ the broadened defect mode $\omega_{0}$, located in the center of the forbidden gap, is filled with $N$ submodes to form a defect band. As was recently reported in Ref. 5, within such a band light propagates at a rather low group velocity and almost without reflection losses, tunneling from one cell to another.

In contrast, in Figs. 4(a) and 4(b) we present transmission spectra of a system consisting of two cavities interacting at a distance that is less than the size of a single cell; one may say that the cavities are mixed rather than coupled [Figs. 1(c) and 1(d)]. Such types of resonators [let us call them mixed-cavity resonators (MCRs)] may be encoded as $(A B)_{J}\left\{D(A B)_{J} D\right\}(A B)_{J}$, where the subscript $J^{\prime}$ may take any value in the range $[1,2 J-1]$. When $J^{\prime}=2 J$, a MCR degenerates into a CCR. Two typical high- $Q$ modes (solid and dashed curves) depicted in Fig. 1 (d) correspond to different eigenfrequencies $\omega_{ \pm}$derived from the original defect mode $\omega_{0}$. Inspection of the $\rho(\omega)$ spectrum reveals that these new mixed-cavity (MC) 


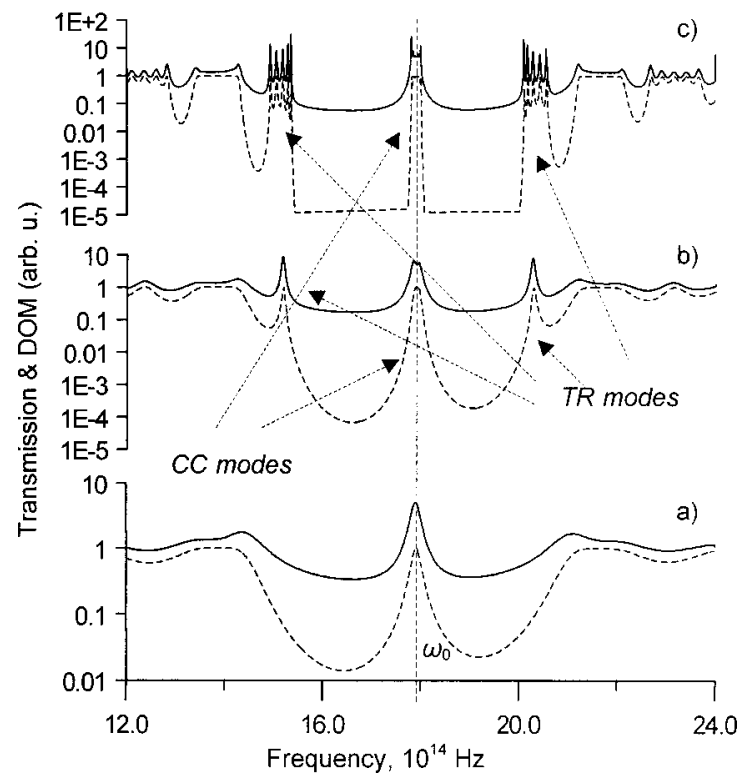

Fig. 3. Transmission and DOM spectra for (a) an $(A B)_{5} D(A B)_{5}$ defect microcavity, (b) $\mathrm{CCR}\left\{(A B)_{5} D(A B)_{5}\right\}_{2}(N=2)$, and (c) CCR $\left\{(A B)_{5} D(A B)_{5}\right\}_{6}(N=6)$. The parameters of the layers are $n_{A}=1.41, d_{A}=0.2, n_{B}=1.0, d_{B}=0.25, n_{D}=1.0, d_{D}$ $=0.25$; there is no optical gain $(g=0)$.

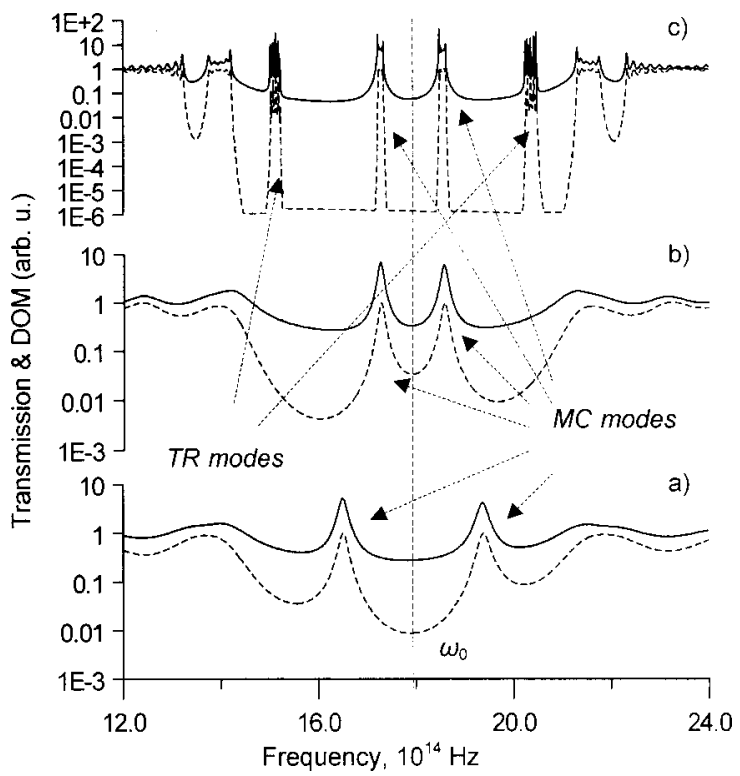

Fig. 4. Transmission (dashed curves) and DOM (solid curves) spectra: (a) MCR $\quad(A B)_{5}\left\{D(A B)_{3} D\right\}(A B)_{5}$; (b) MCR $(A B)_{5}\left\{D(A B)_{5} D\right\}(A B)_{5} ;$ (c) coupled MCR $\left\{(A B)_{5}\left\{D(A B)_{3} D\right\}\right.$ $\left.\times(A B)_{5}\right\}_{6}(M=2, N=6)$. The parameters of the layers are similar to those in Fig. 3; there is no optical gain.

modes are characterized by high values of DOM, which are comparable with the DOM of a single defect microcavity. A fairly remarkable feature is that the structure permits perfect tunneling at $\omega_{ \pm}$. At the same time, the passband at $\omega_{0}$ is replaced with a stop band.

What is most important in the PBG layout being proposed is that, whereas the position of $\omega_{0}$ is determined by the optical parameters (refractive index and thickness) of defect layer $D$, the value of splitting $\Delta \omega=\omega_{ \pm}-\omega_{0}$ de- pends only on parameter $J^{\prime}$. That is, the more closely packed the interacting cavities are in a MCR (the lesser is $J^{\prime}$ ), the larger is $\Delta \omega$ [Figs. $4(\mathrm{a})$ and $\left.4(\mathrm{~b})\right]$. Therefore, flexible control of MCR optical properties is attained at no extra technological cost.

Here we can state with more clarity the difference between CCR and MCR layouts. Until now we estimated coupling between cavities with respect to the distance between defect layers (assuming that the other parameters of PBG material were fixed). In that case the terms "strong" and "weak" coupling did not reflect all nuances. In part, for a small $J$ in an $\left\{(A B)_{J} D(A B)_{J}\right\}_{N}$ CCR structure, the coupling has to be considered strong, and it does cause a significant deviation of frequencies of the generated coupled-cavity submodes. ${ }^{5}$ Nevertheless, the TB limit seems to be an adequate description for this case if one analyzes the wave functions, i.e., intensity distribution $I(z)$, of separate cells. The light pattern in a single unit cell of a CCR looks similar to the pure localized state of a defect microcavity [Figs. 1(b), 1(c), and 1(e)]. That is the reason why, with increasing $N$, these submodes form a wide but continuous passband, whose spectral width does not exceed the bandwidth of a unit cell. However, for an $(A B)_{J}\left\{D(A B)_{J}, D\right\}(A B)_{J}$ MCR, regardless of how large parameter $J$ is, when the condition $J^{\prime} \leqslant 2 J-1$ is true, MC modes are distinctly separated. The increase of $J$ does not influence the splitting $\Delta \omega=\omega_{ \pm}-\omega_{0}$ at fixed $J^{\prime}$ but merely raises the $Q$ factors of modes and narrows their bandwidth. As for the associated intensity distribution [Figs. 1(d) and 1(f)], it is a superposition of localized states of mixed cells. Thus the most distinguishing feature of the various PBG layouts under consideration is the type of localized state.

Now we answer the question: What happens if one constructs a PBG material by translating $N$ times a complex unit cell consisting of two mixed cavities? [An equivalent structure is encoded as $\left\{(A B)_{J}\left\{D(A B)_{J} D\right\}\right.$ $\times(A B)_{J\}_{N}}$; see Fig. 1(f).] Because light tunnels at $\omega_{ \pm}$ with no losses and the TB limit is satisfied for the complex unit cell, no additional splitting of MC modes occurs with the increase of $N$. Instead, we have the effect of formation of perfect transmission bands whose spectral width is equal to the bandwidth of the fundamental MC modes of the complex unit cell [Fig. 4(c)].

Let us analyze the feasibility of such a coupled MCR for laser applications. It is important that, although the DOM within the derived transmission bands is rather high on average, the maximum $\rho$ is attained at the edges of the bands. Hence, no matter how large $N$ is, one attains only doubling of the number of high- $Q$ modes per band. Rather than increasing $N$, another approach to obtaining more low-threshold lasing modes is to increase the number of MCs in a unit cell. In the most general case of a coupled MCR we may write $\left[(A B)_{J}\left\{D(A B)_{J_{1}{ }^{\prime}} D(A B)_{J_{2}} D \ldots(A B)_{J_{M-1}} D\right\}(A B)_{J}\right]_{N}$, where $M$ is the number of microcavities in a coupled MCR, $N$ is the number of translations, and subscripts $J_{1}{ }^{\prime}, J_{2}{ }^{\prime}, \ldots J_{M-1}{ }^{\prime}$ vary within $[1,2 J-1]$. So we can get $M \mathrm{MC}$ bands with $N$ sublevels per band and $2 M$ of high- $Q$ modes among them (Fig. 5).

It is worth now seeking deeper insight into additional high- $Q$ modes, which are inherent in all types of resona- 


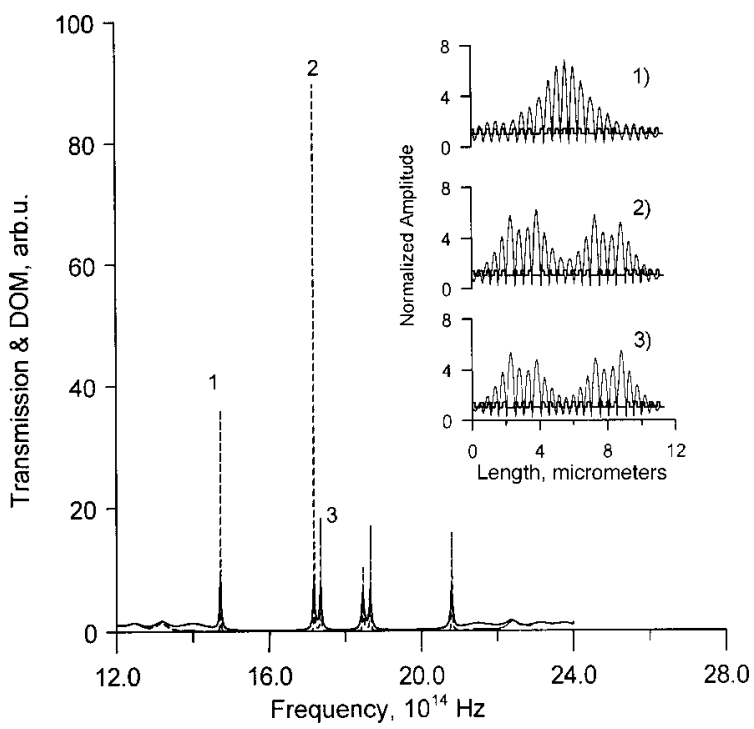

Fig. 5. Transmission (dashed curves) and DOM (solid curves) spectra for an active MCR system containing four cavities, $(A B)_{5}\left\{D(A B)_{3} D(A B)_{7} D(A B)_{3} D\right\}(A B)_{5}$. Insets, $|E(z)|$ for high- $Q$ modes: (1) TR mode, (2), (3) MC modes. The parameters of the layers are similar to those in Fig. 3; the optical gain per layer is $g=0.02 \mu \mathrm{m}^{-1}$.

tors that contain more than one defect layer. These modes arise symmetrically on both sides of the forbidden gap far from its center, and they appear to be the fundamental modes of the internal resonator formed by pairs of coupled cavities. In their intensity distributions these modes are similar to the transmission resonance modes of a generic periodic stack [cf. Fig. 1(a) and the dashed curve in Fig. 1(c)]. Besides, the total amount of modes is equal to $N-1$ for each side of the forbidden gap, just as for transmission resonances. ${ }^{4,8}$ So it is reasonable to call those modes transmission resonance modes (TR modes) (see Figs. 3-5).

\section{DESIGNING THE PHOTONIC STRUCTURES WITH ACTIVE $n-i-p-i$ LAYERS}

We study the lasing properties of semiconductor superlattices embedded in a PBG material. Of possible candidates for active media, $n-i-p-i$ superlattices seem to be the best. They are feasible for integration into optical circuits and, moreover, one can fabricate a crystal in a desirable manner to provide appropriate dispersion and emission properties in a wide spectral range. We propose a realistic design for the new bandgap microcavities developed in Section 3 and apply it for creation of lowthreshold supercompact lasers.

Our generic model of a laser crystal is depicted in Fig. 6. The active layers are made from $n-i-p-i$ superlattices. They are controllable, and their optical properties are modified as a result of electric or optical excitation. The superlattice period is an order of magnitude smaller than the period of the bandgap structure; therefore an approximation of the effective refractive index may be applied for a description of the optical properties of the active medium. The refractive index of $n-i-p-i$ layers depends on the wavelength of light and the difference in the quasi-Fermi levels $\Delta F$.
For constructing a composite bandgap material we suggest use of heterostructures in the GaAs- $\mathrm{Ga}_{x} \mathrm{In}_{1-x} \mathrm{P}$ system, which are lattice matched to the GaAs substrate. Superlattice layers are made as GaAs $n-i-p-i$ crystals with $\delta$-doped $n$ and $p$ layers of 2.8-nm thickness and with $i$ layers of 6.8- or 11.3-nm thickness. Depending on the impurity concentrations and the layer thickness, the effective bandgap of the doping superlattice changes from 0.04 to $0.53 \mathrm{eV}$ as a result of excitation. Accordingly, the refractive indices and the gain spectra vary in a wide range. We have started from the opportunity for growth of bandgap structures in a $\mathrm{GaAs}-\mathrm{Ga}_{x} \operatorname{In}_{1-x} \mathrm{P}$ system and considered the lattice constants of the compounds and lattice matching requirements. Therefore we adjusted the thicknesses of the GaAs $n-i-p-i$ layers to a lattice constant of $0.565 \mathrm{~nm}$ and assumed that $\mathrm{Ga}_{x} \operatorname{In}_{1-x} \mathrm{P}$ layers have the mole fraction of $x=0.51$. Thus the wideband material is $\mathrm{Ga}_{0.51} \mathrm{In}_{0.49} \mathrm{P}$, whose forbidden gap is $1.89 \mathrm{eV}$.

We chose an $n-i-p-i$ superlattice with $n$-doped and $p$-doped layers of thickness $d_{n}=d_{p}=2.83 \mathrm{~nm}, i$ layers of thickness $d_{i}=11.31 \mathrm{~nm}$, and concentrations of donors and acceptors of $N_{d}=N_{a}=5.3 \times 10^{19} \mathrm{~cm}^{-3}$. The total length of the superlattice is $113 \mathrm{~nm}$ and covers four periods. The surface concentration of impurities is 1.5 $\times 10^{13} \mathrm{~cm}^{-2}$, and the effective bandgap of the superlattice decreases to $E_{g}=0.04 \mathrm{eV}$. As can be seen from Fig. 7 , at the proper level of excitation $(\Delta F>1.4 \mathrm{eV})$ the imaginary part of the $n-i-p-i$ layers' refractive index becomes negative, resulting in light amplification within a certain spectral range $(\sim 0.9 \mu \mathrm{m})$.

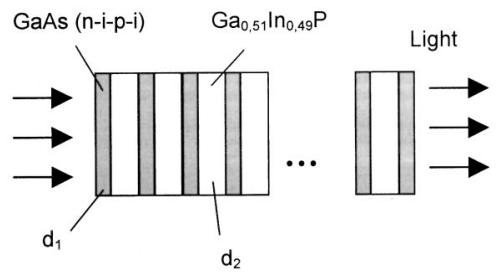

Fig. 6. One-dimensional PBG structure with GaAs $n-i-p-i$ active layers.

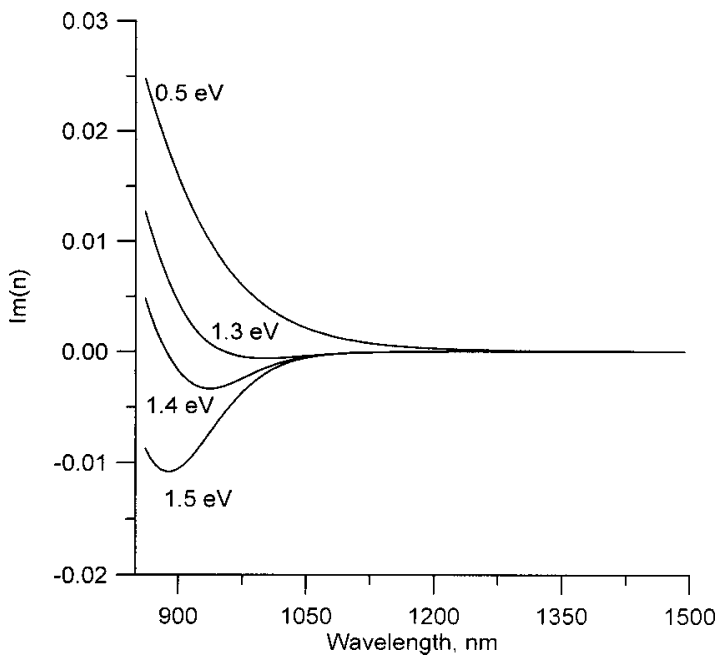

Fig. 7. Imaginary part of the $n-i-p-i$ layers' refractive index versus excitation level $\Delta F$. 

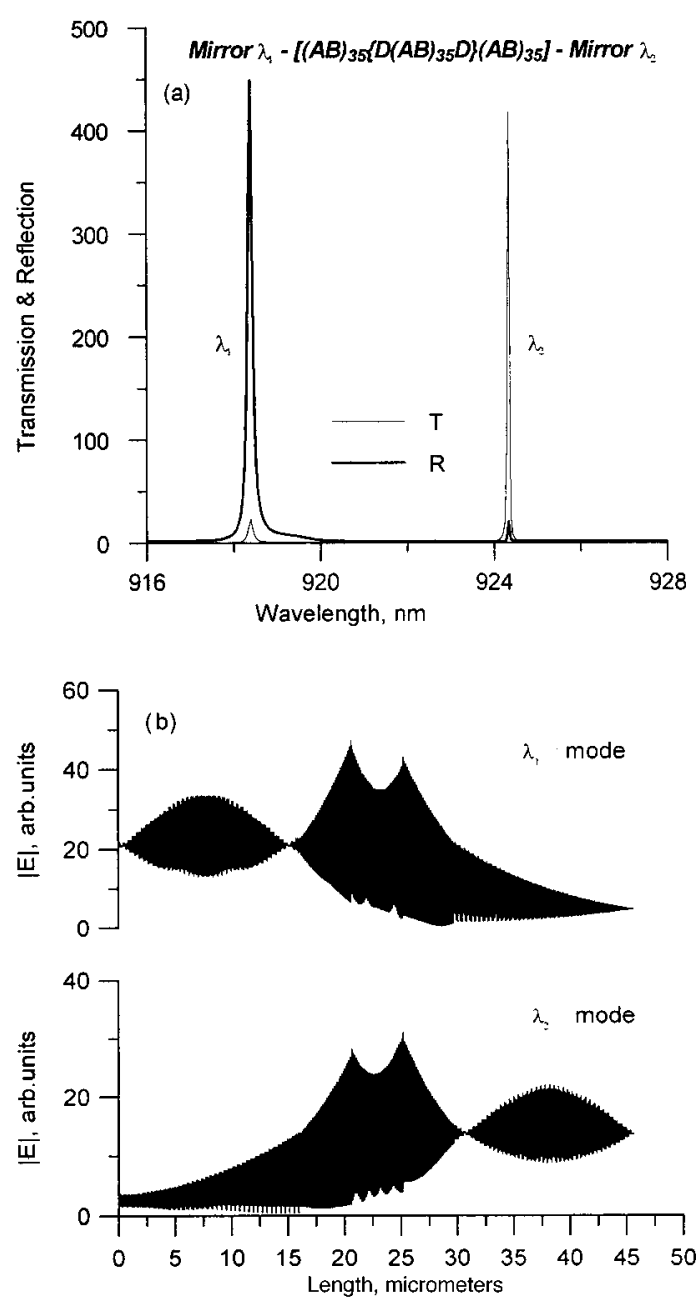

Fig. 8. Lasing PBG MCR structure with active $n-i-p-i$ layers $(\Delta F=1.42 \mathrm{eV}): \quad$ (a) transmission $(\mathrm{T})$ and $(\mathrm{R})$ reflection spectra for two lasing modes, (b) distribution of light amplitude inside the resonator for two different modes.

To attain high lasing efficiency we have to tune our PBG material within the amplification band of an active medium. So we chose the following parameters for the generic $(A B)_{N}$ stack: Layer $\mathrm{A}$ is the $n-i-p-i$ superlattice, and layer $\mathrm{B}$ is the wide-gap semiconductor component $\mathrm{Ga}_{0.51} \mathrm{In}_{0.49} \mathrm{P}$. Because the size of active layers is fixed in our PBG structures, $\mathrm{Ga}_{0.51} \mathrm{In}_{0.49} \mathrm{P}$ layers are given a thickness of $16 \mathrm{~nm}$, which shifts the PBG to the $0.9-\mu \mathrm{m}$ region. The difference between the refractive indices of layers $\mathrm{A}$ and $\mathrm{B}, 2\left(n_{A}-n_{B}\right) /\left(n_{A}+n_{B}\right) \sim 0.06$, is not large, so the total number of layers in the bandgap structures proposed is $\sim 100$.

We consider a model in which a pump uniformly excites the entire structure and $\Delta F$ remains constant within all active layers. Active $n-i-p-i$ superlattice layers can be pumped optically or electrically. Design of the entire photonic structure is similar to a configuration used for vertical-cavity surface-emitting lasers. ${ }^{12}$ Because of the high doping regions of $n-i-p-i$ layers, appropriate selective contacts ${ }^{13}$ provide sufficient low ohmic resistance and efficient injection of nonequilibrium current carriers into the controlling layers of a photonic crystal's heterostructure.
For simplicity it is also assumed that the PBG heterostructure is grown upon an infinite GaAs substrate. In other words, no additional reflection occurs at the end faces of the PBG crystal. Various schemes, for example, antireflection coating and Brewster-angle polishing, are available to make the last-named condition true.

On the basis of the PBG layouts discussed above we propose a 1-D microresonator that is attractive for simultaneous lasing at two wavelengths. The resonator that we present is a system of two mixed microcavities similar to the one described in Fig. 1(d). Then lasing is realized simultaneously at two separate MC modes (wavelengths $\lambda_{1}$ and $\lambda_{2}$ ). The extra feature of this laser system is the possibility that it can guide the radiation that has been generated in different output directions, depending on operating wavelength. The solution is possible owing to the presence of additional selective Bragg mirrors that block the light output at the resonator end faces. The blocking Bragg mirrors can also be constructed in the GaAs- $\mathrm{Ga}_{x} \mathrm{In}_{1-x} \mathrm{P}$ system. We propose the following design: $\quad(G c)_{40}\left[(A B)_{35}\left\{D(A B)_{35} D\right\}(A B)_{35}\right](G C)_{40}$, where $G$ is a GaAs layer of $0.209-\mu \mathrm{m}$ thickness, $\mathrm{C}$ is the $0.188-\mu \mathrm{m} \mathrm{Ga}{ }_{0.51} \mathrm{In}_{0.49} \mathrm{P}$ layer, and $c$ is the $\mathrm{Ga}_{0.51} \mathrm{In}_{0.49} \mathrm{P}$ cell of $0.193-\mu \mathrm{m}$ length. Figure 8 depicts transmission and reflection spectra as well as light patterns for MC modes calculated when the pump is close to lasing threshold. It clearly can be seen that lasing occurs simultaneously in opposite directions, backward at $\lambda_{1}$ and forward at $\lambda_{2}$ wavelength.

\section{SUMMARY}

We have proposed a new, simple, intuitive design for a one-dimensional photonic bandgap material that produces multiple high- $Q$ propagation modes that originate from the fundamental eigenmode of a defect microcavity. The major advantage of the design is that it can produce an arbitrary number of high- $Q$ modes with controllable photon density of modes, bandwidth, and position in the spectrum. Such structures are most prominent for the creation of low-threshold semiconductor lasers operating at multiple wavelengths. Besides, the remarkable feature of the PBG architecture proposed to support perfect transmission of light at low values of group velocity within multiple guiding channels could be applied to the fabrication of multipassband filters and delay lines in optoelectronic circuits and could also be implemented in two- or three-dimensional coupled-cavity waveguides ${ }^{11,14}$ to enrich their multiplexing-demultiplexing ability. Another interesting application is enhanced parametric frequency conversion for ultrashort pulse regimes in upconversion and downconversion laser systems. ${ }^{15,16}$

In addition, we have studied the optical properties of $n-i-p-i$ superlattices embedded into bandgap material. The feasibility of using $n-i-p-i$ heterostructures for laser applications has been shown. New types of laser microcavities are proposed to provide low-threshold lasing at multiple wavelengths and in opposite directions. The integrated assembly of such microresonators pumped either by electric current or optical radiation seems promising for wavelength-division-multiplexing technology. 


\section{ACKNOWLEDGMENTS}

This research was supported by the Belarussian Republican Foundation for Fundamental Research and the Russian Foundation for Basic Research under joint project F99R-119/620.

A. G. Smirnov's e-mail address is andr@dragon.basnet.by

\section{REFERENCES}

1. Yu. Vlasov, K. Luterova, I. Pelant, B. Honerlage, and V. Astratov, "Enhancement of optical gain of semiconductors embedded in three-dimensional photonic crystals," Appl. Phys. Lett. 71, 1616-1618 (1997).

2. J. Dowling, M. Scalora, M. Bloemer, and C. Bowden, "The photonic band edge laser: a new approach to gain enhancement," J. Appl. Phys. 75, 1896-1899 (1994).

3. Y. C. Tsai, K. W. Shumg, and S. C. Gou, "Impurity modes in one-dimensional photonic crystals-analytic approach," J. Mod. Opt. 45, 2147-2157 (1998).

4. M. Scalora, R. Flynn, S. Reinhardt, R. Fork, M. Tocci, M. Bloemer, M. Bowden, H. Ledbetter, J. Bendickson, J. Dowling, and R. Leavitt, "Ultrashort pulse propagation at the photonic band edge: large tunable group delay with minimal distortion and loss," Phys. Rev. E 54, R1078-R1081 (1996).

5. M. Bayindir, S. Tanriseven, and E. Ozbay, "Propagation of light through localized coupled-cavity modes in onedimensional photonic band-gap structures," Appl. Phys. A 72, 117-119 (2001).

6. G. H. Döhler, "Doping superlattices (' $n-i-p-i$ crystals')," IEEE J. Quantum Electron. QE-22, 1682 (1986).
7. V. K. Kononenko, I. S. Manak, and D. V. Ushakov, "Optoelectronic properties and characteristics of doping superlattices," in Photoconversion: Science and Techologies, M. Pluta and M. Szyjer, eds., Proc. SPIE 3580, 10-27 (1998).

8. A. Yariv and P. Yeh, Optical Waves in Crystals (Wiley, New York, 1984)

9. J. M. Bendickson, J. P. Dowling, and M. Scalora, "Analytic expressions for the electromagnetic mode density in finite, one-dimensional, photonic band-gap structures," Phys. Rev. E 53, 4107-4121 (1996).

10. M. Born and E. Wolf, Principles of Optics (Pergamon, Cambridge, 1998)

11. A. Yariv, Y. Xu, R. Lee, and A. Scherer, "Coupled-resonator optical waveguide: a proposal and analysis," Opt. Lett. 24, 711-713 (1999).

12. J. L. Jewel, J. P. Harbison, A. Scherer, Y. H. Lee, and L. T. Florez, "Vertical-cavity surface-emitting lasers: design, growth, fabrication, characterization," IEEE J. Quantum Electron. 27, 1332-1346 (1991)

13. G. H. Döhler, G. Hasnain, and J. N. Miller, "In situ grown-in selective contacts to $n-i-p-i$ doping superlattice crystals using molecular beam epitaxial growth through a shadow mask," Appl. Phys. Lett. 49, 704-706 (1986).

14. Y. Xu, R. Lee, and A. Yariv, "Propagation and secondharmonic generation of electromagnetic waves in a coupledresonator optical waveguide," J. Opt. Soc. Am. B 17, 387 400 (2000).

15. M. Scalora, M. Bloemer, A. Manka, J. Dowling, C. Bowden, R. Viswanathan, and J. Haus, "Pulsed second-harmonic generation in nonlinear, one-dimensional, periodic structures," Phys. Rev. A 56, 3166-3174 (1997).

16. M. Centini, M. Scalora, C. D'Aguanno, C. Sibilia, M. Bertolotti, M. Bloemer, C. Bowden, and J. Haus, "Efficient nonlinear infrared parametric generation in one-dimensional photonic band-gap structures," Opt. Commun. 189, 135142 (2001). 